\title{
ANALYTICAL SOLUTION AND NUMERICAL VERIFICATION FOR THE PRESSURE-RELIEF METHOD OF A CIRCULAR TUNNEL
}

\author{
Shunchuan $\mathrm{Wu}^{1,2}-$ Miaofei $\mathrm{Xu}^{1 *}-$ Yongtao Gao ${ }^{1,2}-$ Shihuai Zhang $^{1}-$ Fan Chen $^{3}$ \\ ${ }^{1}$ The School of Civil and Environment Engineering, University of Science and Technology Beijing, Beijing 100083, \\ China \\ ${ }^{2}$ Key Laboratory of Ministry for Efficient Mining and Safety of Metal Mines, University of Science and Technology \\ Beijing, Beijing 100083, China \\ ${ }^{3}$ Power China Road Bridge Group Co., Ltd., Beijing 100048, China
}

\begin{tabular}{l} 
ARTICLE INFO \\
\hline Article history: \\
Received: 15.06 .2016$. \\
Received in revised form: 14.12 .2016$. \\
Accepted: 16.12 .2016$. \\
\hline Keywords: \\
Pressure relief \\
Complex variable theory \\
FLAC \\
Analytical solution \\
Intersection \\
\hline
\end{tabular}

DOI: https://doi.org/10.30765/er.38.3.11

\section{Introduction}

As an underground structure, a tunnel is widely used in transportation, water conservancy, mining, and it extends horizontally and vertically as human developing commands for resource and space. Putting more emphasis on safety and economy, more emergent but unpredictable failures in rock mass,

\begin{abstract}
:
This paper presents an elastic analytical solution to a circular tunnel with releasing slots at high stress areas near the hole by using a conformal mapping method and the complex variable theory. Compared to the original stress distribution around the circular hole, the releasing effect on elastic stresses is evaluated. After grooving slots, low stress area is generated where the high stress concentration is located. This is agreeable with what was predicted by the finite difference FLAC ${ }^{2 D}$. Besides, displacements are obtained along the periphery of the released hole and are in accordance with those of $F L A C^{2 D}$. In addition to the intersection of the mapping contour, the influences of the sampling points distribution, series number in mapping function, and slot shape are discussed. It is inevitable that the mapping accuracies for the slot and the circle cannot be satisfied at the same time The mapping effect on the circle has to be considered primarily since the stress distribution around the circle is much more significant than the tunnel stability. The analytical solution can be available and fast method of estimating the releasing effect of the application on the tunnel without rock parameters.
\end{abstract}

particularly in high in-situ pressure, result from excavation of activated tunnels. Some soft and sequential failures such as rock spalling or slabbing may be only costly nuisance, but the disruptive ones including rock burst are really dangerous for safety of the construction work force .

A number of researches are carried out about rock spalling or slabbing in circular tunnels under high in

\footnotetext{
* Corresponding author. Tel.: +8615201452188

E-mail address: ustbxmf@163.com
} 
situ stress, and many approaches are also proposed to investigate the brittle fail process in the rock mass near the face of advanced excavation such as iterative elastic analyses (Curran and Corkum [1], Read [2]), strain-soften approach, (Carvalho et al[3]) and constant-deviatoric-stress criterion (Castro [4]). However, fracturing and breakout in rock mass can be attributed to accumulated strain energy relief caused by tunnel excavation when taking no account of their fail process and propagation rules, which was also stated by Martin [5]:One of the major risks associated with the stress-induced spalling in hard rocks is a potential for violent release of stored strain energy.

Aglawe [6] attempted to calculate the released energy of an opening extension simplified as a circle into an ellipse. However, the energy had to be increased with the increasing rock mass quality and this method is only appropriate for sudden failure.

When a rock deteriorates rapidly, in situ pressure increases extremely, and it is inevitable that tunnel or other underground structures are deviated into new profiles from the previous ones. Compared with the self-adaptation and energy natural relief, technologies of rock pressure relief, including distress blasting and distress holes, have actually been an important option of supporting designation that was applied for decades in the underground constructions particularly in mine tunnels. Ortlepp [7] herein considered that the attempt to oppose irresistible movement by increasing strength of support is futile, but proposed that the supporting technologies had the objective of controlling the fracturing and potential disruption of the surrounding rock. For soft rock mass such as coal mine, distress holes or relief slots are applied widely in preventing rock burst in deep coal excavation in Germany, Russia and present China. Ortlepp [8] introduced the application of distress holes with large diameters in preventing potential rock burst in coal tunnels. Bleniawskiat [9] tested the pressure relief extent by using distress holes with different drilling parameters including the drilling diameters and hole pitches. Liu [10] concluded a technological principle by cutting different depth seams along the coal roadway in order to reduce width of coal pillar. Liu [11] carried out a combined supporting with pressure relief boreholes and bolt mesh in a coal tunnel. However, compared to better application of the pressure relief technologies, less studies and verifications about their mechanisms are proposed until numerical simulation is promoted (Liu [12], Sun [13], Song [14]). The availability of numerical methods is obvious in analyzing stress distribution in underground excavation and other engineering problems [15-16], yet some simplifications have to be taken when modeling and analyzing the underground excavation, which lead to a potential uncertainty in assessing the correctness of the simulation. Hence, an analytical solution by the classic methods is still important to promote the assessment, but few researches on the analytical solution of rock pressure relief in tunnel can be found.

For circular holes in two-dimensional elastic planestrain condition, the stress-deformation can be given by Kirsch's elasticity solution [17], but for most noncircular caverns, the complex variable methods proposed by Muskhelishvili [18] have been applied widely. By using the method, Mitchell [19] solved the stress-concentration problem for a doubly symmetrical hole with two circles intersecting the opposite sides of the central one. Similarly, Exadaktylos [20] investigated a semi-analytical elastic solution of the notched circular cylindrical opening in isotropic and homogeneous rock. Based on a conformal mapping function, $\mathrm{Lv}$ [21] illustrated the stress analytical solutions for non-circular holes (e.g. semicircular, three-centered arch, horse-shoe). Zhao [22] found the analytical solution for rock stress around square tunnels in a homogeneous, isotropic, and elastic rock mass by using the complex variable theory. Furthermore, the elastic stress and displacement solutions for tunnels with support were investigated by employing the complex variable method. Savin [23] solved the problem of a circular hole strengthened by an elastic ring, Huo [24] investigated the analytical solution for deep rectangular structures with a far-field shear stress, $\mathrm{Li}$ [25] carried out an elastic plane solution for stresses and displacements around a lined tunnel under in situ stress, Lv[26] derived the stress and displacement field for a horse-shoe supported tunnel subjected to in situ stress based on a conformal transformation method. However, the assumption of homogeneity and isotropy in geomaterial and in situ stress may come into existence at high depth and in high stress. However, the support consideration may not be able to keep a tunnel in practical stability under the circumstance.

The elastic stresses and displacements around a circular hole in isotropic and homogenous rock mass can be carried out by Kirsch solution, and breakouts may probably occur in the region of maximum tangential stress [27], which concentrates on two opposite sides of the boundary [28]. Hence, it is of 
much feasibility to reduce the concentrated stresses by grooving relief slots or drilling boreholes in the high stress zone when the principle stress field in rock mass is known. However, it is difficult to groove even drill boreholes in hard rocks because of the low efficiency and high cost, but it is much easier to implement them in soft rocks technologically.

With the consideration of complexity of stress distribution around non-circular holes (the high stress concentration is effected by hole shape significantly due to the possible cusps). This paper proposes a released circular tunnel by grooving two slots in the region of high stress concentration at tunnel periphery in isotropic and homogenous rock mass and investigates an elastic analytical solution by using a conformal mapping method [29] and the complex variable theory. The maximum principal stress is horizontal, while the minimum principal stress is vertical. By using the solution, the released stress distribution along the hole boundary is given in comparison to the unreleased one obtained by Kirsch solution. Besides, the full-field stresses including tangential and radial stresses along the periphery and $O x$ - and $O y$ - axes, are evaluated when takingthe gradient effect into account. Moreover, the mapping availability of the released hole is discussed. FLAC $^{2 \mathrm{D}}$ is employed to be in comparison with the analytical solution in this paper.

\section{The Analytical Methods}

\subsection{Conformal mapping representation}

For some contours (e.g. circle, ellipse even square), the mapping functions may be simple or investigated previously. As the key of analytical accuracy and solution feasibility, it is of much significance to acquire the mapping function of a certain shape established by a proper method.

For every point $z=x+y \mathrm{i}=r e^{i a}$ in exterior of a simple closed curve in $z$-plane, there exists the corresponding point $\zeta=\xi+\eta \mathrm{i}=\rho e^{i \theta}$ in exterior area of the unit circle in $\zeta$-plane mapped (Fig.1) by the mapping function as Laurent series

$$
z=x+y \mathrm{i}=w(\zeta)=\alpha_{0} \zeta+\sum_{k=1}^{n} \alpha_{2 k-1} \zeta^{-(2 k-1)}
$$

where the constant coefficients $\alpha_{0}, \alpha_{2 k-1}$ $(k=1,2 \ldots, n)$ are real numbers when the loading and geometry configuration are symmetric to both $O x$ and $O y$-axes. The mapping function can be trigonometrically transformed as follows:

$$
\begin{aligned}
& x=\alpha_{0} \rho \cos \theta+\sum_{k=1}^{n} \rho^{-(2 k-1)} \alpha_{2 k-1} \cos (2 k-1) \theta \\
& y=\alpha_{0} \rho \sin \theta-\sum_{k=1}^{n} \rho^{-(2 k-1)} \alpha_{2 k-1} \sin (2 \mathrm{k}-1) \theta
\end{aligned}
$$

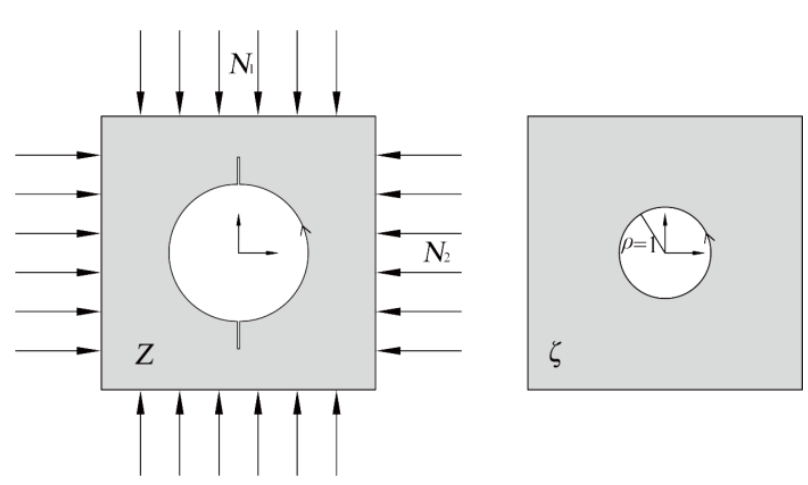

Figure 1. The released hole with doubly symmetric slots in $z$-plane and the unit circle in $\zeta$ plane.

Note that the points describing the contour in $z$-plane and those representing the unit circle in $\zeta$-plane should move in the same direction (anti-clockwise sense is chosen as shown in Fig.1).

There are a number of methods to establish the mapping function such as complex method or Faber series method. Few researches nonetheless investigated the mapping problem of contour with great distortion in limited scale (e.g. the relief slot in this paper). By using the conformal mapping method established by Huangfu [27], the mapping contour and the mapping function can be obtained as follows: (i) The contour line $C$ in $z$-plane is divided into $(m+1)$ sampling points $z_{0}\left(x_{0}, y_{0}\right)$ (the number $m$ and points distribution are determined by the complication and the mapping accuracy requirement). Similarly, $(m+1)$ corresponding points $\zeta_{i}\left(1, \theta_{i}\right)$ need to be represented to the unit circle. In the first approximation, the division of the unit circle can be taken as $\theta=2 \pi / \mathrm{m}$ equidistantly. The constant coefficients $\alpha_{0}, \alpha_{2 k-1}$ $(k=1,2 \ldots, n)$ in the first iteration can be calculated as

$$
\begin{aligned}
& \mathbf{A X}=\mathbf{B} \\
& \mathbf{X}=\left(\mathbf{A}^{\mathrm{T}} \mathbf{A}\right)^{-1} \mathbf{A}^{\mathrm{T}} \mathbf{B}
\end{aligned}
$$


where

$$
\begin{aligned}
& \mathbf{A}=\left[\begin{array}{cccc}
\cos \theta_{0} & \cos \theta_{0} & \cos 3 \theta_{0} & \cdots \\
\cos \theta_{1} & \cos \theta_{1} & \cos 3 \theta_{1} & \cdots \\
\vdots & \vdots & \vdots & \cdots \\
\cos \theta_{m+1} & \cos \theta_{m+1} & \cos 3 \theta_{m+1} & \cdots \\
\sin \theta_{0} & -\sin \theta_{0} & -\sin 3 \theta_{0} & \cdots \\
\sin \theta_{1} & -\sin \theta_{1} & -\sin 3 \theta_{1} & \cdots \\
\vdots & \vdots & \vdots & \cdots \\
\sin \theta_{m+1} & -\sin \theta_{m+1} & -\sin 3 \theta_{m+1} & \cdots
\end{array}\right. \\
& \ldots \quad \cos (2 n-1) \theta_{0} \\
& \text {.. } \cos (2 n-1) \theta_{1} \\
& \text {... } \quad \vdots \\
& \text {... } \cos (2 n-1) \theta_{m+1} \\
& \text {... }-\sin (2 n-1) \theta_{0} \\
& \text {... }-\sin (2 n-1) \theta_{1} \\
& \text {.. } \quad \vdots \\
& \cdots \quad-\sin (2 n-1) \theta_{m+1} \\
& \mathbf{X}=\left[\begin{array}{lllll}
\alpha_{0} & \alpha_{1} & \alpha_{3} & \cdots & \alpha_{2 n-1}
\end{array}\right]^{T} \\
& \mathbf{B}=\left[x_{0}, x_{1}, \cdots, x_{n}, y_{0}, y_{1}, \cdots, y_{n}\right]^{T}
\end{aligned}
$$

(ii) After the first iteration, the new points and their Cartesian coordinates $\mathbf{B}_{1}$ at $z$-plane can be calculated by the first mapping function $z_{1}=w_{1}(\zeta)$, and the distances $l_{i}^{1}$ of every two adjacent new points and their sum $C_{1}$ are also calculated. An assumption herein is taken that the ratios of $l_{i}^{1}$ to $C_{1}$ are equal to those of distances $l_{i}^{1}$ 'of every two adjacent unknown points on the original contour to its known perimeter $C$ as Eq.(5). The Cartesian coordinates of the unknown points are used as new input and substituted into the mapping function. Subsequently, a modified mapping function with new coefficients $\alpha_{0}, \alpha_{2 k-1}$ $(k=1,2 \ldots, n)$ can be established.

$$
\frac{l_{i}^{1}}{C_{1}}=\frac{l_{i}^{1}{ }^{\prime}}{C}
$$

(iii) The iterative procedure is continued until the mapping accuracy is satisfied. The criterion can be as follows

$$
\delta=\frac{\overline{\Delta s}}{C}
$$

Where $\overline{\Delta s}$ is the arithmetic mean value of distances between all mapping points $B_{i}$ and the corresponding points $B_{i}{ }^{\prime}$.

Fig. 2 illustrates approximation of the quarter square boundary with side length of $5 \mathrm{~m}$ ( $\delta=1 \%$ and $n=8$ ). Fig.3 (a) Illustrates the mapping results for notched circular cylindrical opening using the mapping method introduced by Exadaktylos [20] and the method when $n=4$.

Fig.3 (b) Shows the mapping results for the straight wall and semicircle arch using the proposed method and the optimization technique derived by Lv [21] when $n=6$ (densities of sampling points are consistent). It is evident that the mapping accuracies of the proposed method are better than others obtained by the conventional methods. Besides, the proposed method can be programmed more easily, which is of much significance to its application.

Fig. 4 illustrates approximation of the released hole by using the method above with $n=8$ and $n=14$, respectively. There is an intersection at the mapping slot existing in both situations. The cause and effect of the intersection will be discussed below.

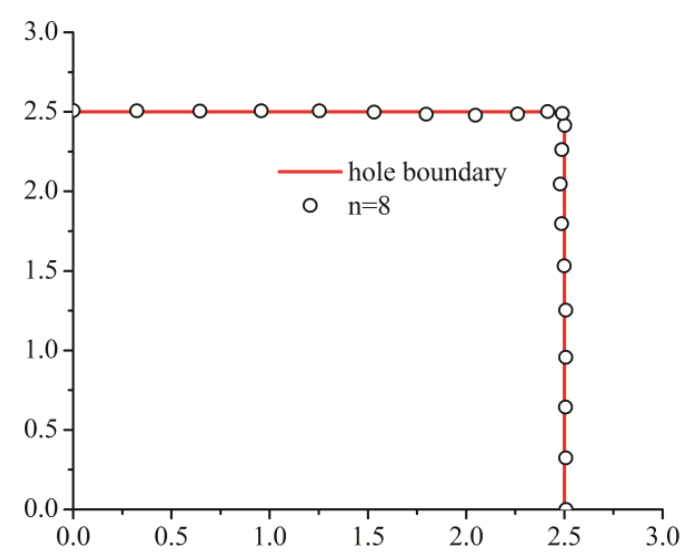

Figure 2. Approximation of the quarter square boundary with length of $5 \mathrm{~m}$.

\subsection{The complex variable theory}

When the mapping function is taken as a finite Laurent series, the potential function can be simplified as Cauchy integral as follows [16]: 


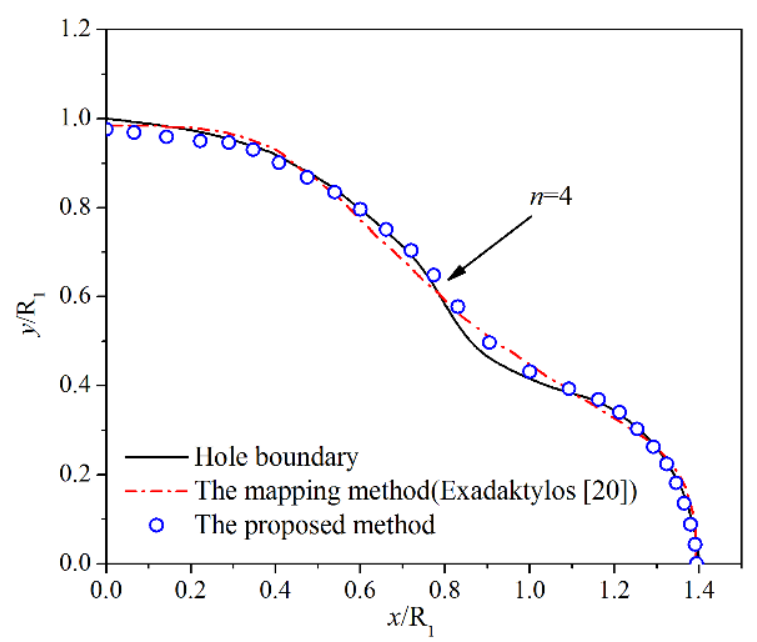

(a)

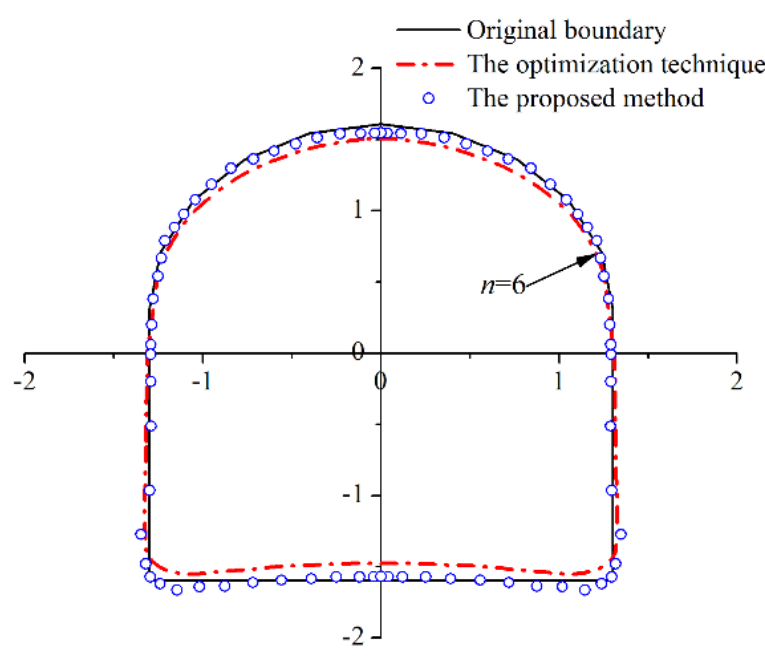

(b)

Figure 3. The comparison of the mapping results between the conventional method and the proposed mapping method: (a) The quarter boundary of notched circular cylindrical opening $\left(R_{1}\right.$ is a radius of circular opening); (b) Straight wall and semicircle arch.

$$
\begin{aligned}
& -\varphi_{0}(\zeta)+\frac{1}{2 \pi i} \int_{\Gamma} \frac{w(\sigma)}{\overline{w^{\prime}(\sigma)}} \frac{\overline{\varphi_{0}{ }^{\prime}(\sigma)}}{\sigma-\zeta}= \\
& =\frac{1}{2 \pi i} \int_{\Gamma} \frac{f(\sigma)}{\sigma-\zeta} d \sigma \\
& f(\sigma)=-2 B w(\sigma)-\left(B^{\prime}-i C^{\prime}\right) \overline{w(\sigma)} \\
& B=\frac{N_{1}+N_{2}}{4} \\
& B^{\prime}=-\frac{N_{1}-N_{2}}{2} e^{-2 i \alpha} \\
& C^{\prime}=0
\end{aligned}
$$

where $w(\sigma)$ and $\overline{w(\sigma)}$ are boundary values of $w(\zeta)$ and $\bar{\omega}\left(\frac{1}{\zeta}\right)$ respectively, $\alpha$ is the angle between the maximum principle stress and horizontal direction $\left(0 \leq \alpha \leq 90^{\circ}\right)$.

By conjugating Eq. (7), Eq. (9) is obtained as

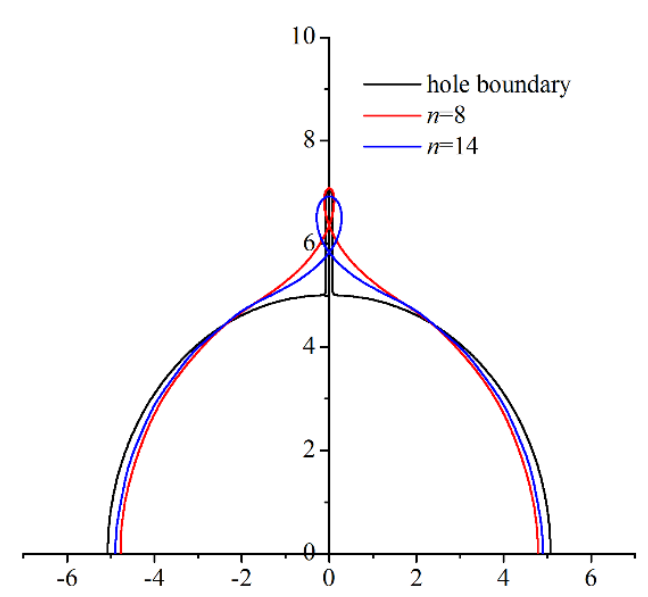

$$
\begin{aligned}
-\psi_{0}(\zeta) & +\frac{1}{2 \pi i} \int_{\Gamma} \frac{\overline{w(\sigma)}}{w^{\prime}(\sigma)} \frac{\varphi_{0}{ }^{\prime}(\sigma)}{\sigma-\zeta}= \\
= & \frac{1}{2 \pi i} \int_{\Gamma} \frac{\overline{f(\sigma)}}{\sigma-\zeta} d \sigma
\end{aligned}
$$

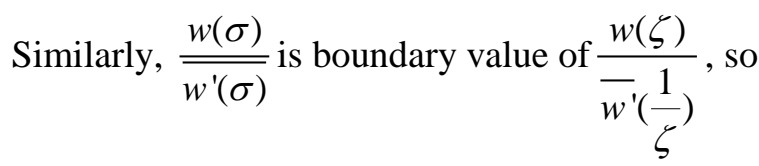

$$
\begin{aligned}
& \frac{w(\sigma)}{\overline{w^{\prime}(\sigma)}}= b_{2 n-1} \sigma^{-2 n-1}+b_{2 n-3} \sigma^{-2 n-3}+ \\
&+b_{2 n-5} \sigma^{-2 n-5}+\cdots+b_{1} \sigma^{-1}+b_{-1} \sigma^{1}+ \\
&+\sum_{k=1}^{\infty} b^{\prime}{ }_{2 k-1} \sigma^{2 k+1}
\end{aligned}
$$


wheretheconstant coefficients $b_{2 k-1}(k=0, \cdots n)$ and $b^{\prime}{ }_{2 k-1}(k=1, \cdots \infty)$ can be represented by $\alpha_{0}$ and $\alpha_{2 k-1}(k=1, \cdots n)$ as:

$$
\begin{aligned}
& b_{2 n-1}=\frac{\alpha_{2 n-1}}{\alpha_{0}} \\
& b_{2 n-3}=\frac{\alpha_{2 n-3}+\alpha_{1} b_{2 n-1}}{\alpha_{0}} \\
& b_{2 n-5}=\frac{\alpha_{2 n-5}+\alpha_{1} b_{2 n-3}+3 \alpha_{3} b_{2 n-1}}{\alpha_{0}} \\
& \ldots \\
& b_{-1}=\frac{\alpha_{0}+\alpha_{1} b_{1}+3 \alpha_{3} b_{3}+5 \alpha_{5} b_{5}+\cdots+(2 n-1) \alpha_{2 n-1} b_{2 n-1}}{\alpha_{0}}
\end{aligned}
$$

Actually, it is insignificant for solving $\varphi_{0}(\zeta)$ to calculate $\sum_{k=1}^{\infty} b_{2 k-1}^{\prime} \sigma^{2 k+1}$, which will be shown below. $\varphi_{0}(\zeta)$ can also be represented as a finite series as Eq. (12).

$$
\varphi_{0}(\zeta)=\sum_{k=1}^{n} c_{2 k-1} \zeta^{-2 k-1}
$$

where $c_{2 k-1}(k=1, \cdots n)$ are the unknown constant real coefficients.

$\frac{w(\sigma)}{\overline{w^{\prime}(\sigma)}} \overline{\varphi_{0}{ }^{\prime}(\sigma)}$ can be expressed as

$$
\begin{aligned}
& \left(b_{2 n-1} \sigma^{-2 n-1}+b_{2 n-3} \sigma^{-2 n-3}+b_{2 n-5} \sigma^{-2 n-5}+\right. \\
& \left.+\cdots+b_{1} \sigma^{-1}+b_{-1} \sigma^{1}+\sum_{k=1}^{\infty} b_{2 k-1} \sigma^{2 k+1}\right) . \\
& \cdot\left[\sum_{k=1}^{n}(-2 k-1) c_{2 k-1} \sigma^{2 k+2}\right] \\
& =\sum_{k=1}^{n-1} K_{2 k-1} \sigma^{-(2 k-1)}+\sum_{k=1}^{\infty} K_{2 k-1}^{\prime} \sigma^{(2 k-1)}
\end{aligned}
$$

the constant real coefficients $K_{2 k-1}(k=1, \cdots n-1)$ canberepresentedby $\quad b_{2 k-1}(k=0, \cdots n) \quad$ and $c_{2 k-1}(k=1, \cdots n)$.

$$
\begin{aligned}
& K_{2 n-3}=-b_{2 n-1} c_{1} \\
& K_{2 n-5}=-b_{2 n-3} c_{1}-3 b_{2 n-1} c_{3} \\
& K_{2 n-7}=-b_{2 n-5} c_{1}-3 b_{2 n-3} c_{3}-5 b_{2 n-1} c_{5} \\
& \cdots \\
& K_{1}=-b_{3} c_{1}-3 b_{5} c_{3}-5 b_{7} c_{5}- \\
& -\cdots-(2 n-3) b_{2 n-1} c_{2 n-1}
\end{aligned}
$$

According to the mapping rule of exterior-exterior in the two planes, $\sum_{k=1}^{\infty} K_{2 k-1}^{\prime} \sigma^{2 k-1}$ can be omitted as an interior analytical solution, due to the positive powers of all monomials in $\overline{\varphi_{0}{ }^{\prime}(\sigma)}, \sum_{k=1}^{\infty} b^{\prime}{ }_{2 k-1} \sigma^{2 k+1}$ does not contribute to calculation of $\sum_{k=1}^{n-1} K_{2 k-1} \sigma^{-(2 k-1)}$ and hence is not essential to be solved.

By substituting Eq. (8), (12) and (13) into (7), the Cauchy integral can be represented as

$$
\begin{aligned}
& -\sum_{k=1}^{n} c_{2 k-1} \zeta^{-(2 k-1)}+\sum_{k=1}^{n-1} K_{2 k-1} \zeta^{-(2 k-1)} \\
& =2 B w(\zeta)-2 B \alpha_{0} \zeta+B^{\prime} \alpha_{0} \zeta^{-1}
\end{aligned}
$$

By comparing the same power of $\zeta$, Eq. (15) can be expressed in matrix form as

$$
\begin{aligned}
& {\left[\begin{array}{ccccc}
-1+b_{3} & 3 b_{5} & \cdots & (2 n-3) b_{2 n-1} & 0 \\
b_{5} & -1+3 b_{7} & \cdots & 0 & 0 \\
b_{7} & 3 b_{9} & \cdots & 0 & 0 \\
\vdots & \vdots & \ddots & \vdots & \vdots \\
0 & 0 & 0 & \cdots & -1
\end{array}\right] } \\
& {\left[\begin{array}{c}
c_{1} \\
c_{3} \\
c_{5} \\
\vdots \\
c_{2 n-1}
\end{array}\right] }=\left[\begin{array}{c}
2 B \alpha_{1}+\alpha_{0} B^{\prime} \\
2 B \alpha_{3} \\
2 B \alpha_{5} \\
\vdots \\
2 B \alpha_{2 n-1}
\end{array}\right]
\end{aligned}
$$

The solution of the linear algebra Eq. (15) for the constant coefficients $c_{2 k-1}(k=1, \cdots n)$ gives the first complex potential function as:

$$
\varphi(\zeta)=B w(\zeta)+\varphi_{0}(\zeta)
$$


By conjugating Eq. (15)

$$
\begin{aligned}
& \psi_{0}(\zeta)=-\left[\frac{\bar{w}\left(\frac{1}{\zeta}\right)}{w^{\prime}(\zeta)} \varphi_{0}^{\prime}(\zeta)-\sum_{k=1}^{n-1} K_{2 k-1} \zeta^{2 k-1}\right]- \\
& -2 B \alpha_{0} \zeta^{-1}-B^{\prime} w(\zeta)+B^{\prime} \alpha_{0} \zeta
\end{aligned}
$$

where

$$
\begin{aligned}
& \bar{w}\left(\frac{1}{\zeta}\right)=\frac{\alpha_{0}}{\zeta}+\sum_{k=1}^{n} \alpha_{2 k-1} \zeta^{2 k-1} \\
& w^{\prime}(\zeta)=\alpha_{0}-(2 k-1) \sum_{k=1}^{n} \alpha_{2 k-1} \zeta^{-2 k}
\end{aligned}
$$

The second complex potential function can be obtained as

$$
\begin{gathered}
\psi(\zeta)=-B^{\prime} w(\zeta)+\psi_{0}(\zeta) \\
=-\left[\begin{array}{c}
\frac{\bar{w}\left(\frac{1}{\zeta}\right)}{w^{\prime}(\zeta)} \varphi_{0}{ }^{\prime}(\zeta)-\sum_{k=1}^{n-1} K_{2 k-1} \zeta^{2 k-1} \\
-2 B \alpha_{0} \zeta^{-1}+B^{\prime} \alpha_{0} \zeta
\end{array}\right]-
\end{gathered}
$$

Hence, $\sigma_{r}, \sigma_{\theta}, \tau_{r \theta} u_{x}, u_{y}$ can be computed by Eq. (21).

$$
\begin{aligned}
& \sigma_{r}+\sigma_{\theta}=4 \operatorname{Re}\left[\frac{\varphi^{\prime}(\zeta)}{w^{\prime}(\zeta)}\right] \\
& \sigma_{\theta}-\sigma_{\rho}+2 i \tau_{r \theta}= \\
& =\frac{2 \zeta^{2}}{\rho^{2}} \frac{1}{\overline{w^{\prime}(\zeta)}}\left\{\overline{w(\zeta)}\left[\frac{\varphi^{\prime}(\zeta)}{w^{\prime}(\zeta)}\right]+\psi^{\prime}(\zeta)\right\} \\
& u_{x}+\mathrm{i} u_{y}=\frac{1}{2 G}\left[\begin{array}{l}
\left.\kappa \varphi(\zeta)-\frac{w(\zeta)}{\overline{w^{\prime}(\zeta)}} \overline{\varphi^{\prime}(\zeta)}\right] \\
-\psi(\zeta)
\end{array}\right]
\end{aligned}
$$

where $G=\frac{E}{2(1+2 \mu)}, \kappa=3-4 \mu$ in plane strain problem.

\section{The results}

In this section, a circular hole with radius of $5 \mathrm{~m}$ is investigated, and two symmetric pressure-relief slots are used at top and bottom of the hole with $N_{1}=15$ $\mathrm{MPa}$ and $N_{2}=9 \mathrm{MPa}$ The depth and width of the slot are $2 \mathrm{~m}$ and $0.14 \mathrm{~m}$, respectively. Meanwhile, the released stress distribution around the released hole is compared with the unreleased result obtained by Kirsch solution without support (Eq. (22)).

$$
\begin{aligned}
& \sigma_{r}=\frac{1}{2}\left(N_{1}+N_{2}\right)\left(1-\frac{R^{2}}{r^{2}}\right)+ \\
& +\frac{1}{2}\left(N_{1}-N_{2}\right)\left(1-4 \frac{R^{2}}{r^{2}}+3 \frac{R^{4}}{r^{4}}\right) \cos 2 \theta \\
& \sigma_{\theta}=\frac{1}{2}\left(N_{1}+N_{2}\right)\left(1+\frac{R^{2}}{r^{2}}\right)- \\
& -\frac{1}{2}\left(N_{1}-N_{2}\right)\left(1+3 \frac{R^{4}}{r^{4}}\right) \cos 2 \theta \\
& \tau_{r \theta}=-\frac{1}{2}\left(N_{1}-N_{2}\right) . \\
& \cdot\left(1+2 \frac{R^{2}}{r^{2}}-3 \frac{R^{4}}{r^{4}}\right) \sin 2 \theta
\end{aligned}
$$

A finite difference model (Fig.5 (a) and (b)) established by the FLAC ${ }^{2 \mathrm{D}}$ is also used to verify the analytical solution.

Fig.6 (a) illustrates $\sigma_{\theta}$ along the peripheries of the circle and the released hole (the slot boundary is also considered). Compared with $\sigma_{\theta}$ of Kirsch solution, the released stress variation shows that there is a superposition with the Kirsch solution result in $\theta<0.6$ It then increases when $0.6<\theta<1$ until it reaches the local maximum at $\theta=1$. After that a sharp decrease is taken in $1<\theta<1.4$, then a rapid increase occurs when $1.5<\theta<\pi / 2$. It is of much significance that the tangential stress of the analytical solution is almost less than that of the Kirsch solution in the scale except $1.5<\theta<\pi / 2$. Moreover, Fig.6 (b) represents the hoop stress along the periphery of the doubly symmetric hole [18], which shows similar regularity with $\sigma_{\theta}$ variation of the released hole in reversal of $\theta$ in Fig.6 (a). Besides, $\sigma_{\theta}$ variation of the analytical solution is in accordance with that of FLAC showed in Fig.6 (a).

Due to the stress-gradient effect, stress distribution in rock mass along the tunnel depth is of great significance for stability of rock mass [25]. 


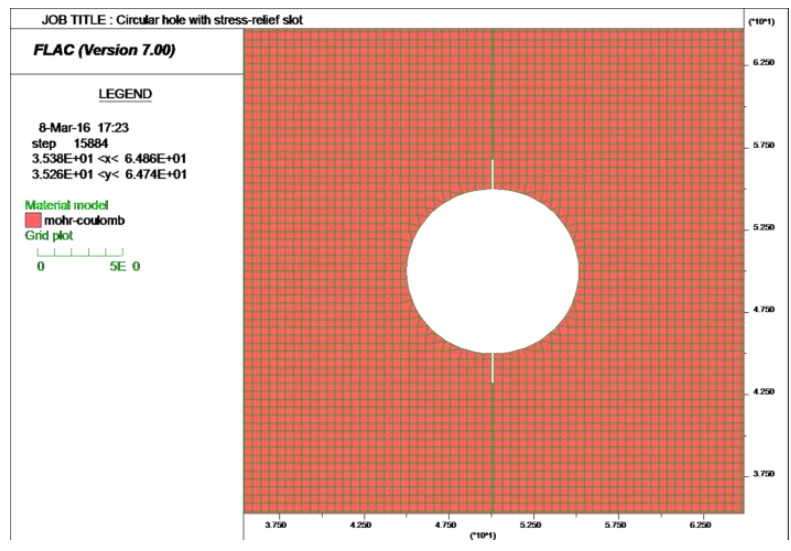

(a)

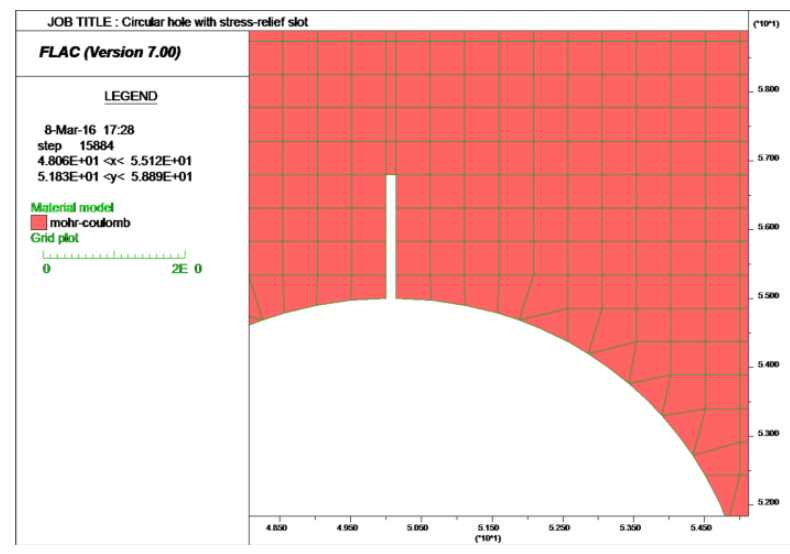

(b)

Figure 5. The FLAC ${ }^{2 D}$ finite difference model for the released hole.

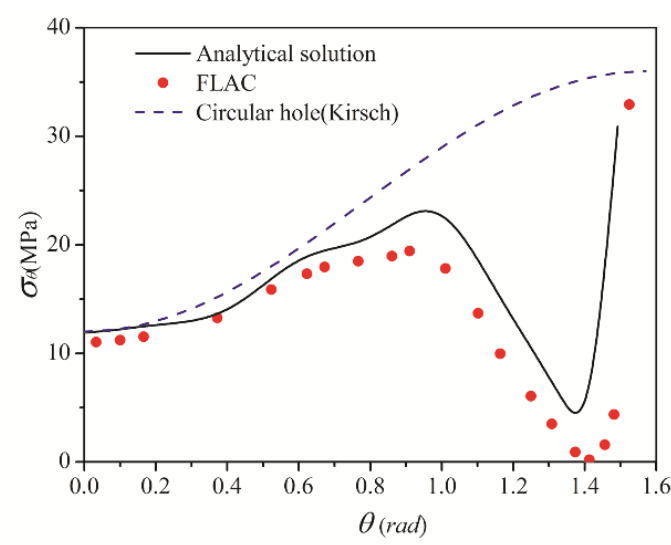

(a)

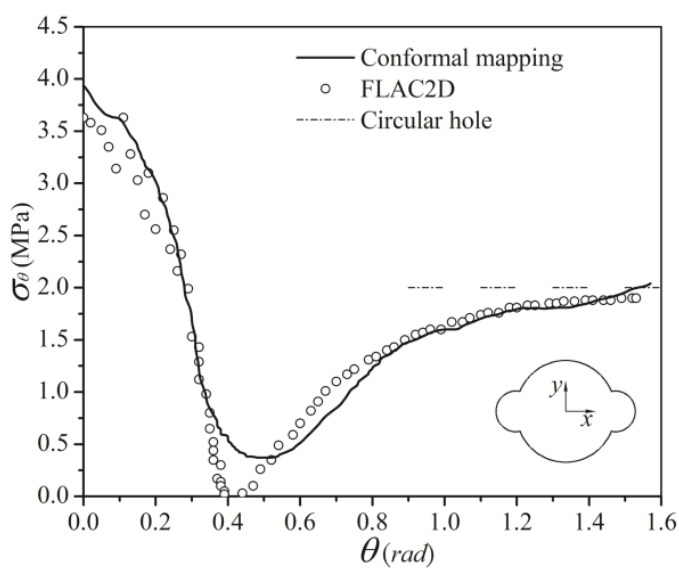

(b)

Figure 6. Comparison of $\sigma_{\theta}$ along the periphery and the circular hole: (a) The released hole; (b) The doubly symmetric hole.

Fig. 7 illustrates comparison of $\sigma_{\theta}$ and $\sigma_{r}$ along $O x$ axis $(\theta=0)$ for the released hole and the circle. Correspondingly, this paper alsocompares stresses along the $O y$-axis $(\theta=\pi / 2)$ in Fig.8.

Fig. 9 presents a comparison of displacement variations along the released hole boundary obtained by the analytical solution and FLAC $^{2 \mathrm{D}}$ ( $E=1 G P a$, $\mu=0.2$ ). The results are in good agreement.

\section{Discussion}

\subsection{The intersection of mapping contour}

In this paper, an intersection on the mapped contour exists, which is significant to correctness of the analytical solution. According to the conformal mapping theory, the mapping efficiency may be influenced by density of the sampling points and series number. Besides, the mapping availability may also be affected by the contour shape in geometry, so the slot shape has to be considered. In this paper, the three factors above are investigated. 


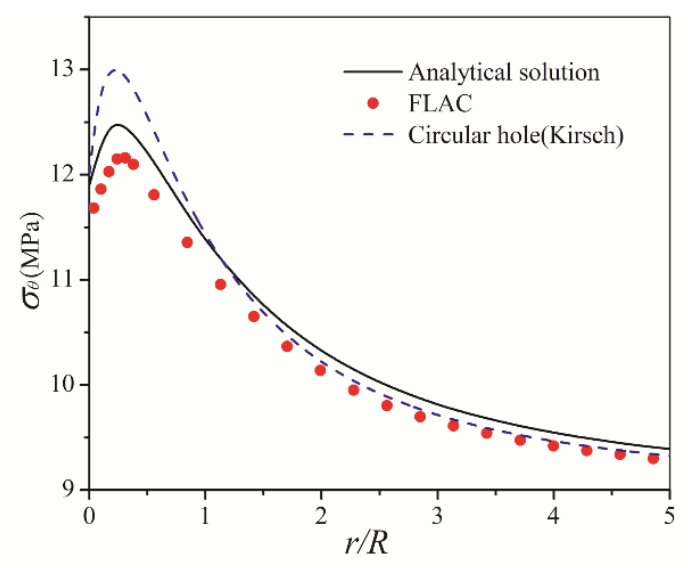

(a)

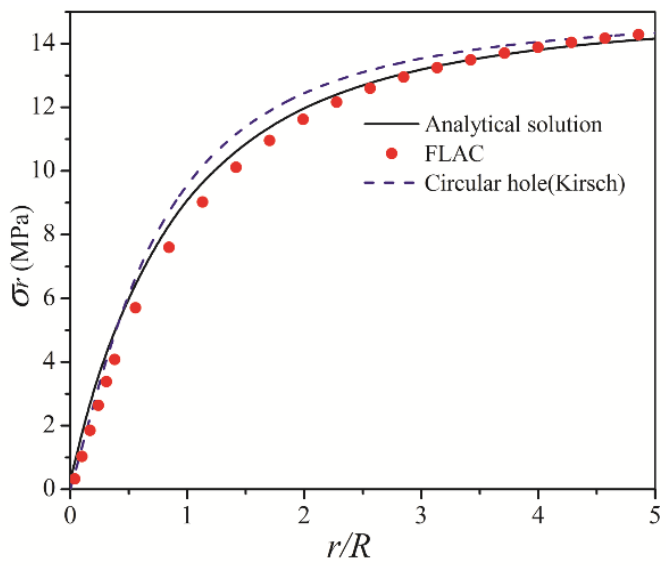

(b)

Figure 7. Comparison of the released hole and the circular hole along $O x$-axis ( $\theta=0)$ : (a) Tangential stress; (b) Radial stress.

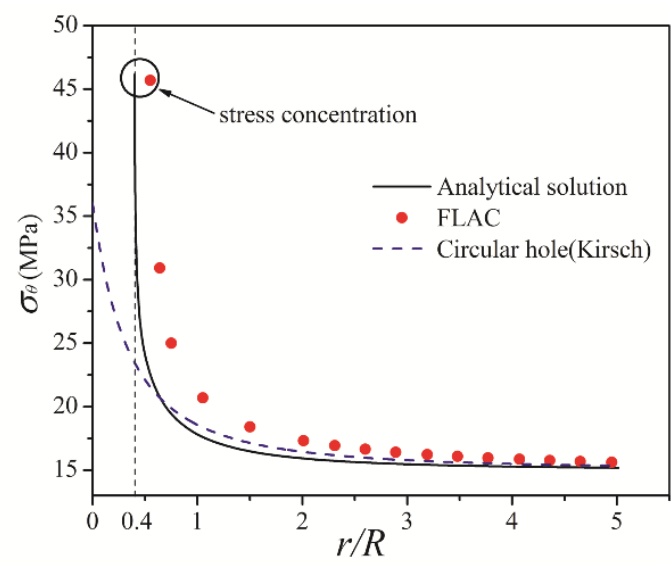

(a)

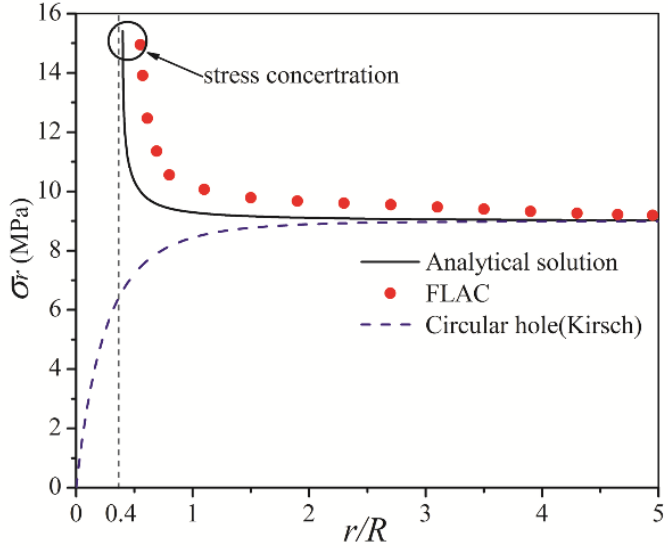

(b)

Figure 8. Comparison of the released hole and the circular hole along Oy-axis $(\theta=\pi / 2)$ : (a) Tangential stress; (b) Radial stress.

(i) The slots adjacent to the hole are independent of the circular part geometrically, so when sampling the points, $n_{1} / n_{2}$ is taken to estimate the effect of density of sampling points on the mapping contour (the original contour is given in Fig.4, $n=8$ ), as shown in Fig.10.The variations of tangential stress with different $n_{1} / n_{2}$ along periphery of the released hole are given in Fig.11.

As is shown in Fig.10, teardrop at top of the contour is mapped for the slot with low $n_{1} / n_{2}$, and it shrinks by increasing $n_{1} / n_{2}$ and disappears when $n_{1} / n_{2}=0.4$. This can be attributed to the increasing sampling points for the slot as those representing the circular part decreasing reversely.

However, it is also apparent that the circular contour is rougher with increasing $n_{1} / n_{2}$, which may lead to a deviant variation in stress distribution as showed in Fig. $10\left(N_{1}=15 \mathrm{MPa}\right.$ and $\left.N_{2}=9 \mathrm{MPa}\right)$. Compared with the monotone increase with $n_{1} / n_{2}=0.08$, humping curves are acquired with $n_{1} / n_{2} \geq 0.2$ in $\theta<0.8$. By calculating angles of the bulges at periphery in the polar coordinates, for instance, the angles $\theta$ are about $26^{\circ}$ and $50^{\circ}$ respectively ( $\theta=0$ at $O x$-axis) with $n_{1} / n_{2}=0.4$, which is in good accordance with the radians (near 0.45 and 0.85) of the peaks in Fig.11. 
However, the hump is of great discrepancy with the FLAC results. In fact, the stress variation around the circular part $(\theta \leq 1.4)$, rather than that of the slot, is more significant in evaluating the tunnel stability.

(ii) It is understandable that series number in the mapping function has significant influence on availability of the method. Fig.12 enumerates the mapping contours with different series numbers. As the series number $n$ increases, the mapping availability in circular part generally improves, the intersection is arising but not being vanished, and the teardrop extends horizontally. Hence, even though the increasing series number contributes to accuracy of the circle, it deteriorates the availability of the relief slot and is not beneficial to eliminate the intersection.

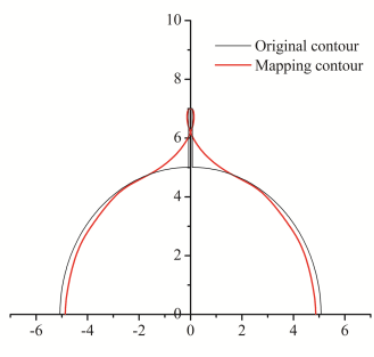

(a)

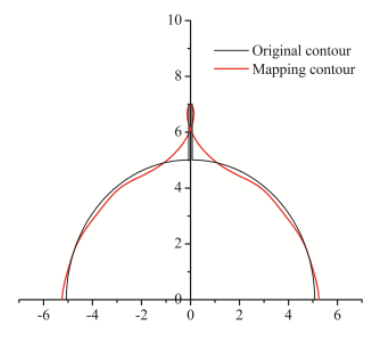

(b)

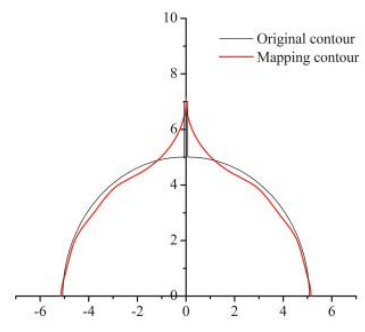

(c)

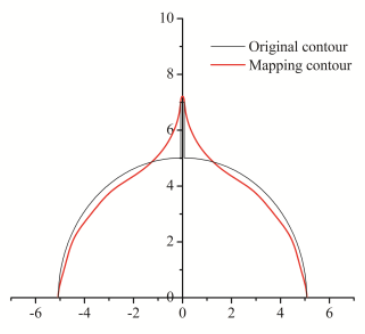

(d)

Figure 10. Approximation of the mapping contours of the released hole: (a) $n_{1} / n_{2}=0.08$; ( $b$ ) $n_{1} / n_{2}=0.2$; (c) $n_{1} / n_{2}=0.4 ;(d) n_{1} / n_{2}=0.6$.

(iii) There is no intersection produced in the known noncircular contours including rectangle, semicircle even the notched circle by conformal mapping method, so shape of the thin slot has to be considered as restriction for approximating availability. Fig. 13 illustrates approximations of the released holes with different $w / h$ of slots $\left(n_{1} / n_{2}=0.2\right)$.It is conspicuous that the teardrop diminishes by increasing $w / h$, and even transforming into cusp when $w / h=0.4$, which implies that the contour with more regular geometry may be mapped easier by the mapping method. Fig.14 illustrates approximation of $\sigma_{\theta}$ along the periphery of released holes with differen $w / h$ thevariations of $\sigma_{\theta}$ and $\sigma_{r}$ along $O x$-axis are shown in Fig. 15 and Fig.16, respectively $\left(N_{1}=15 \mathrm{MPa}\right.$ and $N_{2}=9 \mathrm{MPa}$ ). It is apparent that increasing of $w / h$ has no significant effect on the releasing effect since all the stress curves are intertwined.

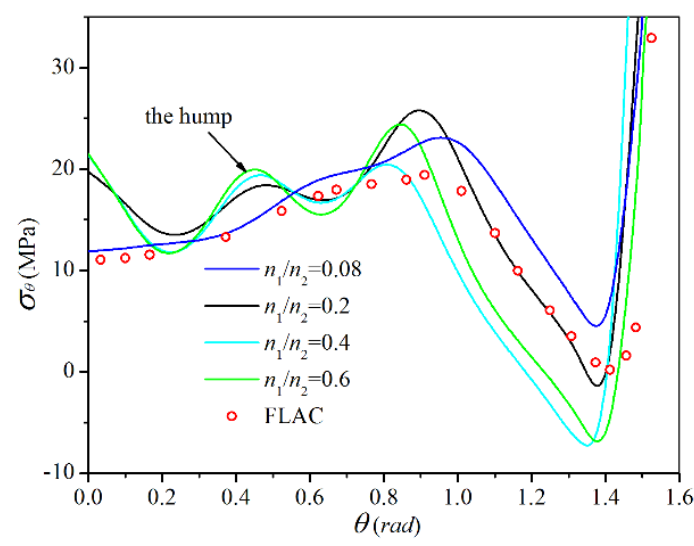

Figure 11. Variations of tangential stresses along the released hole periphery with different $n_{1} / n_{2}\left(N_{1}=15 \mathrm{MPa}\right.$ and $\left.N_{2}=9 \mathrm{MPa}\right)$. 


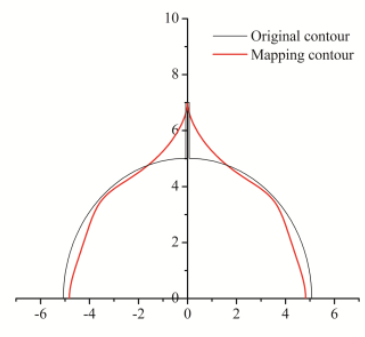

(a)

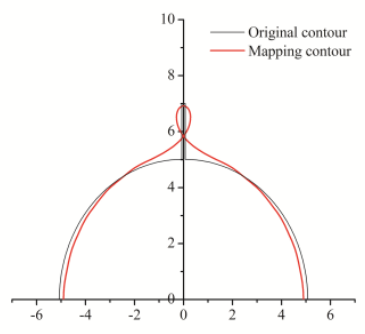

(b)

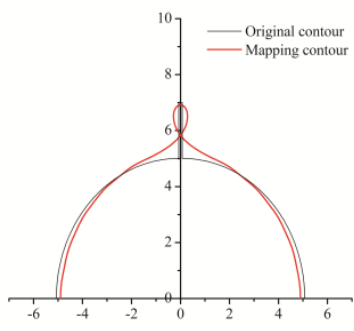

(c)

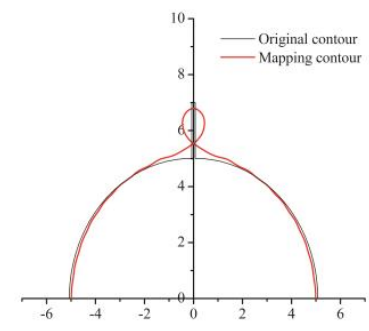

(d)

Figure 12. Approximation of the mapping contours of the released hole: (a) $n=5 ;$ (b) $n=8 ;(c) n=14 ;(d) n=20$.

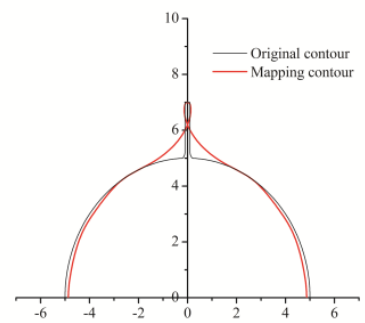

(a)

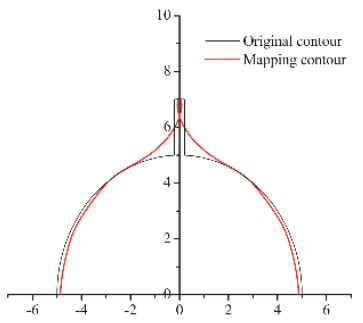

(b)

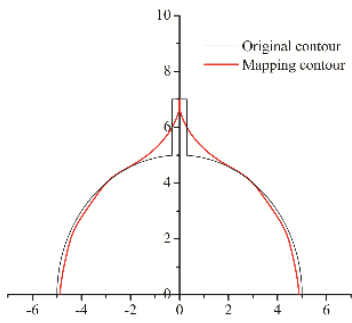

(c)

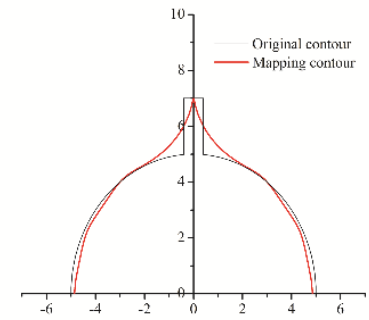

(d)

Figure 13. Approximation of the mapping contours of the released hole $\left(n_{1} / n_{2}=0.2\right):(a) w / h=0.1 ;(b) w / h=0.2$; (c) $w / h=0.3 ;(d) w / h=0.4$.

In fact, it is impossible to take a wide slot technologically and economically in practical construction $(w=0.8 \mathrm{~m}$ in Fig.13 (d)). In this paper, the width of the slot recommendedis mainly only $0.14 \mathrm{~m}$.

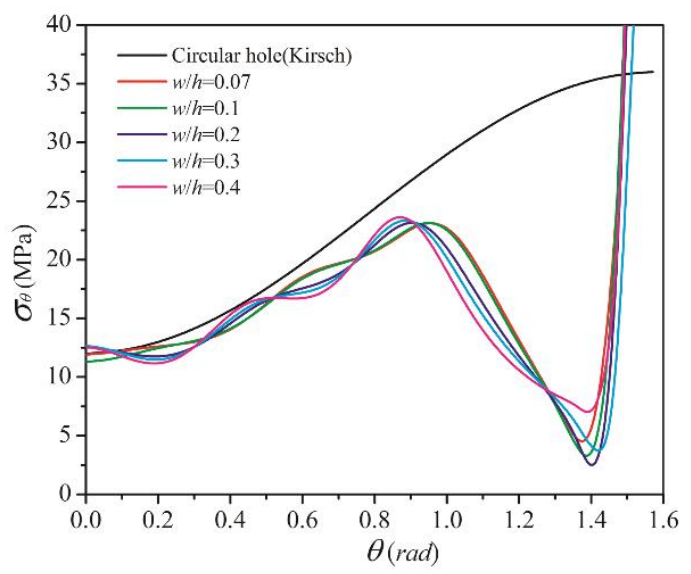

Figure 14. Approximation of the tangential stress along the released holes periphery $\left(N_{l}=15 \mathrm{MPa}\right.$ and $\left.N_{2}=9 \mathrm{MPa}\right)$.

\subsection{Elastic stress distribution}

As is shown in Fig.6 (a) and Fig.14, there is a rigid decrease of tangential stress along the periphery of the released hole when $\theta>1$. In other words, low stress area substitutes for high stress concentration after pressure releasing. However, this effect on stress distribution may decay as the distance to the slot increases, which is proved by the stress variations agreement of the analytical solution and Kirsch solution in scope of $\theta<0.6$ in Fig.6 (a). Besides, the sharp increase of $\sigma_{\theta}$ can be attributed after the previous decrease to the stress transfer caused by the relief slot. The maximum $\sigma_{\theta}$ obtained by the analytical solution and the simulation are 30.891MPa and 36.924MPa respectively in Fig.6 (a), which are close to $36 \mathrm{MPa}$ calculated by the Kirsch solution $\left(\sigma_{\theta}=3 N_{1}-N_{2}\right)$ [30]. However, the maximum is transferred into the rock mass with depth of $2 \mathrm{~m}(r / R=0.4$ in Fig. 8$)$.

In addition, it is also obvious that different releasing effects on $\sigma_{\theta}$ and $\sigma_{r}$ are produced as shown in

Fig.7 and Fig.8.

The peak stresses are generated at $r / R=0.3$ along the $O x$-axis in both the unreleased hole and released 
hole because of the lateral pressure coefficient in Fig.7 (a). However, the peak of the released hole is lower due to the pressure relief effect andin spite of this, the effect decreases rapidly when the depth in rock mass increases. As a result of the analytical solution, simulation and the Kirsch solution are in approximation when $r / R>1$ in Fig.7 (a) and Fig.8 (a). By contrast, there is a lower effect on $\sigma_{r}$ as a good agreement between the released and unreleased stresses along the $O x$-axis. Moreover, the sharp decrease of $\sigma_{r}$ in Fig.8 also proves the rapid collapse of releasing effects by increasing the depth. Besides, there is great stress concentration at

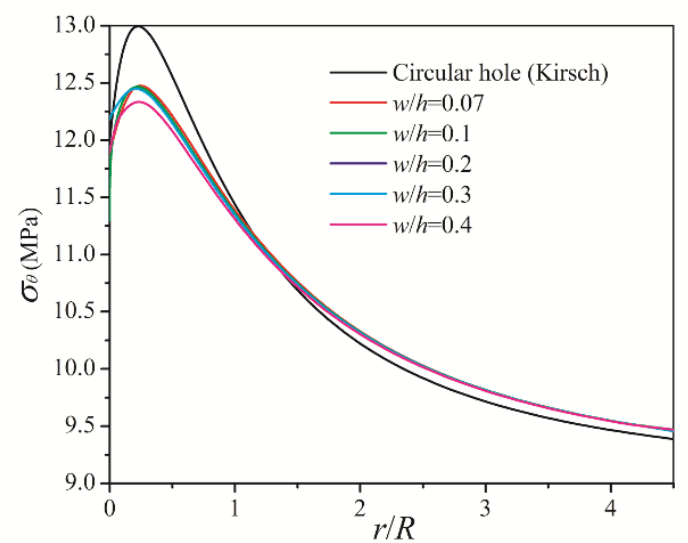

(a) top of the teardrop, which may leads to overlarge stress (e.g. $\sigma_{\theta}=184.31 \mathrm{MPa}$ ) and is not illustrated in Fig.6 (a) since it is beyond the scale. The stress concentration is also the reason of high radial stresses proved by the analytical solution and FLAC.

Fig.16 illustrates the contours of $\sigma_{\theta}$ and $\sigma_{r}$ around the released hole with $w / h=0.07\left(N_{1}=15 \mathrm{MPa}\right.$ and $\left.N_{2}=9 \mathrm{MPa}\right)$. There are distressed zones on both sides of the teardrop, but a high stress concentration is also generated at its top and may lead to yield and fail in rock mass in plastic situation.

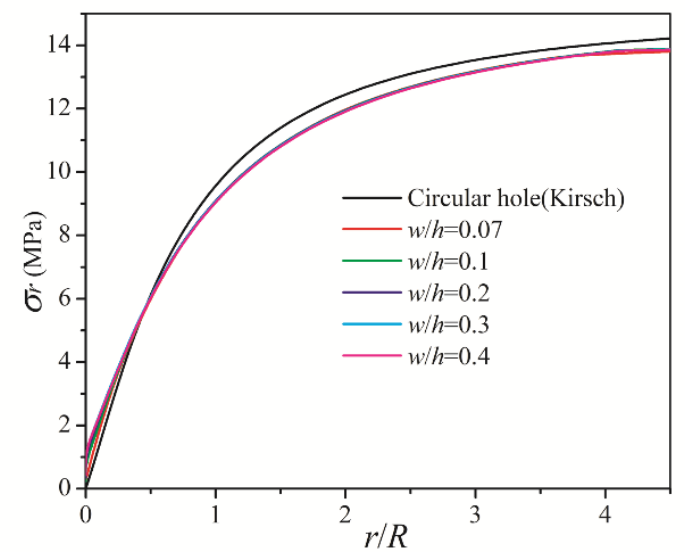

(b)

Figure 15. Approximation of stresses along the Ox -axis $\left(N_{1}=15 \mathrm{MPa}\right.$ and $\left.\mathrm{N}_{2}=9 \mathrm{MPa}\right):($ a)Tangential stress; (b) Radial stress.

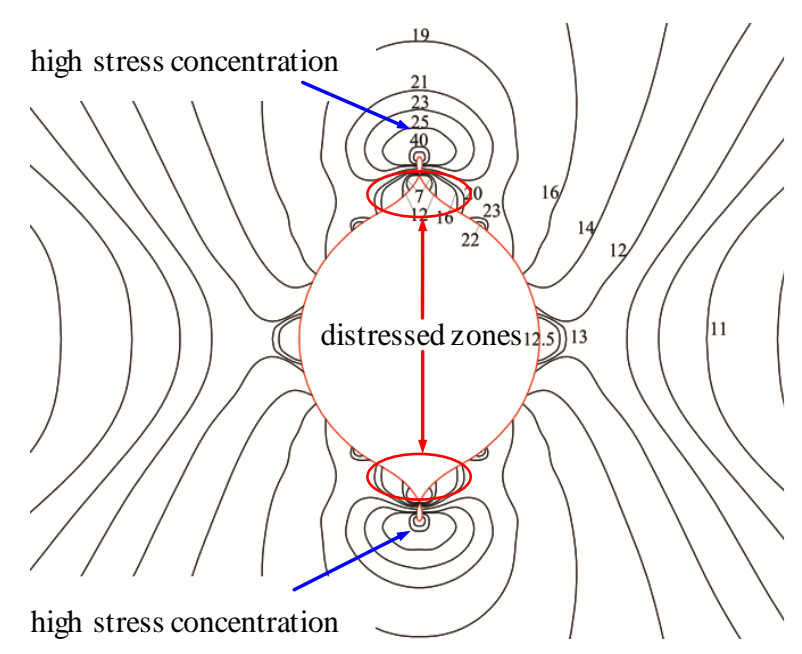

(a)

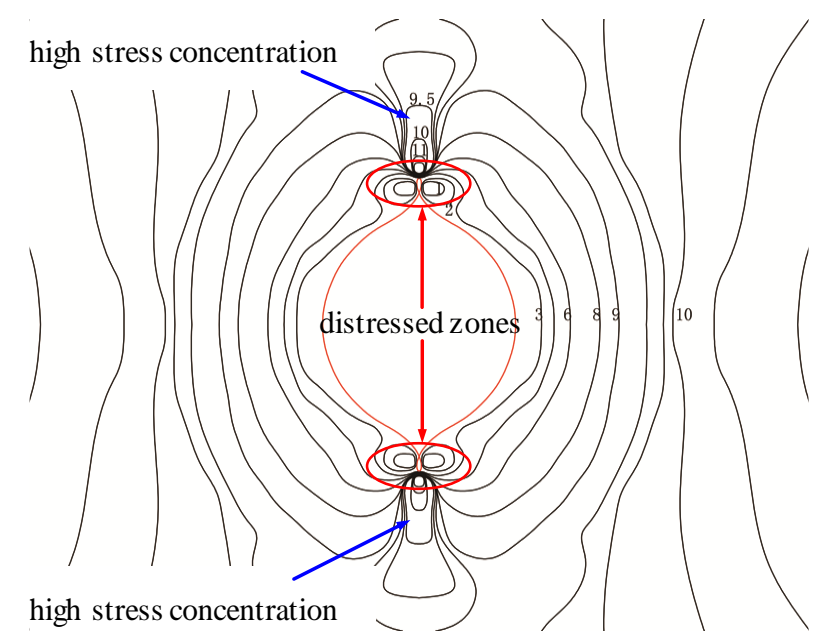

(b)

Figure 17. Stress contours around the released hole $\left(N_{l}=15 \mathrm{MPa}\right.$ and $\left.N_{2}=9 \mathrm{MPa}\right):(a)$ Tangential stress; (b) Radial stress 


\section{Conclusion}

(1) By using a conformal mapping method and the complex variable theory, an elastic analytical solution to circular tunnel with releasing slot was studied in isotropic and homogenous rock masses. FLAC $^{2 D}$ was used in this paper to verify the reasonability of the analytical results.

(2) The tangential stress $\sigma_{\theta}$ along the periphery of the released hole is lower than that of the Kirsch solution. Two low stress areas adjacent to the slot were developed, while the high stress area was transferred into the rock mass by the releasing effect. (3) The releasing effect decays rapidly when increasing distance from the hole boundary to far field.Compared with the $\sigma_{\theta}$, the effect upon $\sigma_{r}$ is lower.

(4) When increasing the sampling points for slot, the intersection of the mapping contour may disappear with less mapping accuracy of the circular part. Increasing the number of terms in mapping function is merely beneficial to mapping accuracy of the circular part, but it does not contribute to elimination of the intersection. Besides, it is easier to acquire the mapping contour without intersection for wider slot, but increasing the width of the slot is not beneficial for enhancing the releasing effect.

\section{Acknowledgement}

The precious financial support by Beijing Training Project for the Leading Talent in S\&T (Z151100000315014) is gratefully acknowledged. In addition, sincere gratitude is expressed for the language modification comments by Jennifer Liang, School of Foreign Languages, University of Science and Technology Beijing.

\section{Nomenclature}

$(r, \alpha)$, the polar coordinates in $z$-plane;

$(x, y)$, the Cartesian coordinates in $z$-plane;

$(\rho, \theta)$, the polar coordinates in $\zeta$-plane;

$(\xi, \eta)$, the Cartesian coordinates in $\zeta$-plane;

$m+1$, number of sampling points;

$n$, series number of mapping function;

$C$, perimeter of the original contour;

$N_{1}$, the horizontal maximum principle stress;

$N_{2}$, the vertical minimum principle stress;

$R$, radius of tunnel; $c$, cohesion;

$\varphi$, friction angle;

$E$, elastic modulus;

$\mu$, Poisson ratio;

$G$, shear modulus;

$\sigma_{r}, \sigma_{\theta}, \tau_{r \theta}$, tangential, Radial and Shear stress;

$u_{x}, u_{y}, x$-displacement and $y$-displacement;

$w(\zeta)$, mapping function;

$\bar{w}\left(\frac{1}{\zeta}\right)$, conjugation of mapping function;

$\varphi(\zeta)$, the first complex potential function;

$\psi(\zeta)$, the second complex potential function;

$w$, slot width;

$h$, slot depth;

$n_{2}$, number of sampling points for circle;

$a_{p}$, distance of the tunnel center to plastic zone

boundary.

\section{References}

[1] Curran, J. H., Corkum, B. T.: Examine2D-a 2D boundary element program for calculating stresses around underground excavations in rock. Rock Eng Group, University of Toronto, Toronto, Ont, 1995.

[2] Read, R. S.: Interpreting excavation-induced displacements around a tunnel in highly stressed granite, Ph.D. thesis, Department of Civil and Geological Engineering, University of Manitoba, Winnipeg, Man, 1994.

[3] Carvalho, J. L., Hoek E., Corkum, B. T.: PHASES-a 2D hybrid finite/boundary element program for calculating stresses and estimating support around underground openings, version 2. Rock Eng. Group, University of Toronto, Toronto, Ont. 1991.

[4] Castro, L. A. M., Mccreath D. R., Oliver, P.: Rock mass damage initiation around the Sudbury Neutrino Observatory cavern. 2nd North American Rock Mechanics Symposium, American Rock Mechanics Association, 1996.

[5] Martin, C. D., Kaiser, P. K., Christianson, R.: Stress, instability and design of underground excavations, International Journal of Rock Mechanics and Mining Sciences, 40(2003)7, 1027-1047.

[6] Aglawe, J. P.: Unstable and violent failure around underground openings in highly stressed 
ground. Diss. Queen's University at Kingston, 1999.

[7] Ortlepp, W. D.: The behavior of tunnels at great depth under large static and dynamic pressures, Tunnelling and Underground Space Technology, 16(2001)1, 41-48.

[8] Ortlepp, W. D., Stacey, T. R.: Rockburst mechanisms in tunnels and shafts, Tunnelling and Underground Space Technology, 9(1994)1, 59-65.

[9] Bleniawskiat, Denkhaushg, Vogleruw: Failure of Fracture Rock, International Journal of Rock Mechanics and Mining Sciences, (1969)6, 323334.

[10] Liu, Z. H., Yang, L. S., Song, X. M. et al.: Stress control of deep cutting along roadway over roof rock, Journal of Mining \& Safety Engineering, 31(2014)3, 347-353.(in Chinese)

[11] Liu, H. G., He, Y. N., Han, L. J. et al.: Numerical simulation and industrial test for combined support of pressure relief boreholes with bolt mesh in deep coal tunnel. Journal of Mining \& Safety Engineering, 23(2006)3, 258-263.(in Chinese)

[12] Liu, Z. H., Zhao, Y. S., Gong, P. L. et al. Distribution characteristics of coal pillar stress after the roadway roof being large depth cutting seam, Journal of China Coal Society, 36(2011)1, 18-23.(in Chinese)

[13] Sun, J., Wang, L. G.: Numerical simulation of grooving method for floor heave control in soft rock roadway, Mining Science and Technology (China), 21(2011)1, 49-56.

[14] Song, X. X., Zuo, Y. J. Wang, X.: Numerical simulation of pressure-released hole combined support with rockbolt in deep roadway with dynamic disturbance. Journal of Central South University (Science and Technology), 45(2014)9, 3158-3165.(in Chinese)

[15] Škec, L., Jelanović, A., Jelenić, G.: Glued timber-concrete beams-analytical and numerical models for assessment of composite action, Engineering Review, 33(2013)1: 41-49.

[16] Jelenković, T., Travaš, V.: Numerical and experimental analysis of seepage beneath a model of a gravity dam, Engineering Review, 33(2013)2: 75-84.

[17] Kirsch, G: Die theorie der elastizität und die bedürfnisse der festigkeitslehre, Springer, 1898.

[18] Muskhelishvili, N. I.: Some basic problems of the mathematical theory of elasticity, Springer Science \& Business Media. 2013.
[19] Mitchell, L. H.: Stress-concentration factors at a doubly-symmetric hole, The Aeronaut, Quart, (1966)17,177-186.

[20] Exadaktylos, G. E., Liolios, P. A., Stavropoulou, M. C.: A semi-analytical elastic stressdisplacement solution for notched circular openings in rocks, International Journal of Solids and Structures, 40(2003)5, 1165-1187.

[21] Lv, A. Z., Zhang, L. Q.: Complex function method on mechanical analysis of underground tunnel. Beijing, China, 2007.

[22] Zhao, G. P., Yang, S. Q.: Analytical solutions for rock stress around square tunnels using complex variable theory. International Journal of Rock Mechanics and Mining Sciences, (2015)80, 302307.

[23] Savin, G. N.: Stress concentration around holes. Pergamon Press, Oxford, 1961.

[24] Huo, H., Bobet, A., Fernandez, G. et al.: Analytical solution for deep rectangular structures subjected to far-field shear stresses, Tunnelling and underground space technology, 21(2006)6, 613-625.

[25] Li, S. C., Wang, M. B.: An elastic stressdisplacement solution for a lined tunnel at great depth, International Journal of Rock Mechanics and Mining Sciences, 45(2008)4, 486-494.

[26] Lv ,A. Z., Zhang, N., Lin, K.: Analytic solutions of stress and displacement for a non-circular tunnel at great depth including support delay. International Journal of Rock Mechanics and Mining Sciences, (2014)70, 69-81.

[27] Jaeger, J. C., Cook, N. G. W.: Fundamentals of rock mechanics, 3rd Chapman and Hall, London, 1979.

[28] Dammyr, Ø.: Prediction of Brittle Failure for TBM Tunnels in Anisotropic Rock: A Case Study from Northern Norway, Rock Mechanics and Rock Engineering, 2016, 1-23.

[29] Huangfu, P. P., Wu, F. Q., Guo, S. F. et al.: A new method for calculating mapping function of external area of cavern with arbitrary shape based on searching points on boundary, Rock and soil Mechanics, 32(2011)5, 1418-24.(in Chinese)

[30] Martin, C. D.: Seventeenth Canadian Geotechnical Colloquium The effect of cohesion loss and stress path on brittle rock strength. Can. Geotech. J. (1997)34, 698-725. 